\title{
POTENCIAL DENDROCRONOLÓGICO DE Juniperus monticola MARTÍNEZ EN EL MONTE TLÁLOC, MÉXICO
}

\section{DENDROCHRONOLOGIC POTENTIAL OF Juniperus monticola MARTÍNEZ IN MOUNTAIN TLÁLOC, MÉXICO}

\author{
José Villanueva-Díaz', Lorenzo Vázquez-Selem² ${ }^{1}$ Armando Gómez-Guerrero ${ }^{3 *}$, \\ Julián Cerano-Paredes ${ }^{1}$, Noé A. Aguirre-González ${ }^{4}$ y Osvaldo Franco-Ramos ${ }^{2}$
}

\begin{abstract}
'CENID RASPA, Instituto Nacional de Investigaciones Forestales, Agrícolas y Pecuarias (INIFAP). Km 6.5 Margen Derecha del Canal Sacramento. Gómez Palacio. 35140, Gómez Palacio, Durango, Dgo. nstituto de Geografía, Universidad Nacional Autónoma de México. Ciudad Universitaria. 04510, México, CDMX. ${ }^{3}$ Postgrado en Ciencias Forestales, Colegio de Postgraduados. Km 36.5 Carr. México-Texcoco, Montecillo-Chapingo. 56230, Estado de México, México. ${ }^{4}$ Instituto de Ciencias Agropecuarias y Rurales, Universidad Autónoma del Estado de México. Km. 14.5 Carr. Toluca-Atlacomulco. 50000, Toluca, Edo. Mex.
\end{abstract}

*Autor para Correspondencia (agomezg@colpos.mx)

\section{RESUMEN}

Se desarrolló una cronología de anillo anual de 264 años (1750 a 2013) con 33 series derivadas de 50 especímenes de Juniperus monticola Martínez (enebro, cedro blanco) del Monte Tláloc, en un intervalo altitudinal de 3980 a $4060 \mathrm{~m}$. A pesar de la irregularidad de las secciones transversales y de la variabilidad en el ancho del anillo anual, los parámetros dendrocronológicos de COFECHA y ARSTAN como sensibilidad media (0.41), inter-correlación entre series (0.55) y relación señal-ruido (1.24) indicaron que la especie es viable para estudios dendrocronológicos. La correlación de la serie cronológica con la temperatura media anual fue estadísticamente significativa $(r=0.45, n=$ $54, P<0.01)$, y también se encontró una correlación significativa $(P<0.01)$ con series fechadas de Pinus hartwegii de los volcanes Iztaccíhuatl y Pico de Orizaba, lo que confirma su potencial dendrocronológico. Asimismo, se obtuvieron correlaciones significativas con índices $\mathrm{SOI}(\mathrm{r}=-0.27, \mathrm{P}<0.01, \mathrm{n}$ $=113)$ y con el PDSI reconstruido para junio en el centro de México $(r=0.25$, $P<0.01, n=109$ ), lo que es una prueba de la factibilidad para interpretar fenómenos climáticos a partir de anillos de enebro en altitudes elevadas (> $3500 \mathrm{msnm}$ ) del centro de México. Para la especie estudiada la temperatura media fue la variable más correlacionada con su crecimiento radial. Los años intensos de El Niño, que producen sequías severas en el centro de México, se asociaron con mayores incrementos radiales. El enebro es idóneo para realizar estudios de la influencia de este fenómeno y para comprender la variabilidad climática histórica de alta y baja frecuencia.

Palabras clave: Crecimiento radial, enebro mexicano, ENSO, Juniperus monticola.

\section{SUMMARY}

A 264-year chronological study (1750-2013) for total ring width was produced from 33 series of 50 specimens of Mexican juniper MJ, (Juniperus monticola Martínez), also known as enebro or cedro blanco, at Mount Tláloc, along an altitudinal range from 3980 to 4060 masl. Despite irregular transversal sections and ring-width variability of this species, COFECHA and ARSTAN dendrochronological parameters like mean sensitivity $(0.41)$, series intercorrelation (0.51), and signal-to-noise ratio (1.24) indicated that for dendrochronological studies are viable on this species. The correlation of the ring width series to mean annual temperature was statistically significant $(r$ $=0.45, n=54, p<0.01$ ). Also, a significant correlation was found for other dated series in Pinus hartwegii from the Iztaccíhuatl and Pico de Orizaba volcanoes. Significant correlations were found between the $S O I(r=-0.27, p$
$<0.01, \mathrm{n}=113$ ) and PDSI indexes reconstructed for June in Central México $(r=0.25, p<0.01, n=109)$. These results indicate that climatological events could be reliably predicted from rings of MJ trees grown at high altitudes (> $3500 \mathrm{msnm}$ ). For this species, mean annual temperature correlated highest to ring width. Intense El Niño events associated to high radial growth. Given human influence on climate change and the ecological impact on high altitude species, MJ is a reliable species to understand climatic variability.

Index words: Radial growth, Mexican juniper, ENSO, Juniperus monticola.

\section{INTRODUCCIÓN}

México es un país megadiverso con enorme potencial dendrocronológico, situación que se ha demostrado mediante el desarrollo de más de 170 series de crecimiento fechadas (Villanueva et al., 2011). Sin embargo, este avance en la integración de una red dendrocronológica para México es limitado, ya que solo el género Pinus supera las 50 especies (Farjon et al., 1997). Las series dendrocronológicas generadas hasta ahora han sido de gran utilidad para entender la variabilidad interanual y multianual de variables climáticas de los últimos 500 años para el norte y centro de México (Villanueva et al., 2011; Correa-Díaz et al., 2014; Villanueva et al., 2014a), así como para cuantificar la influencia que ejercen fenómenos circulatorios como El Niño Oscilación del Sur (ENSO, por sus siglas en inglés) (Seager et al., 2009; Stahle et al., 2012) y la Oscilación Decadal del Pacífico (PDO, por sus siglas en inglés) (Biondi et al., 2001).

La mayoría de las especies que se han estudiado en México, son sensibles a la precipitación y a los cambios de ritmo de crecimiento de primavera e invierno (Díaz et al., 2001; Brito-Castillo et al. 2003; Villanueva et al., 2009a; Pompa-García et al., 2015); sin embargo, la correlación del crecimiento con temperatura es menos frecuente en 
especies forestales, aunque en algunos estudios locales se han encontrado correlaciones significativas (CorreaDíaz et al., 2014). Las evidencias de mayor crecimiento por aumento en la temperatura en árboles del límite altitudinal arbóreo ayudan a comprender el efecto de cambio climático en los bosques (Álvarez et al., 2015).

Actualmente, debido a la falta de información histórica hay incertidumbre sobre el impacto del incremento en la temperatura en la dinámica y sobrevivencia de especies arbóreas. Dada la escasa distribución de estaciones meteorológicas en elevaciones mayores a 3000 msnm y la menor variabilidad climática de las montañas elevadas, las series de anillos anuales de especies del límite altitudinal arbóreo son las más indicadas para comprender los impactos de cambio climático (Grace et al., 2002). Los estudios dendrocronológicos a más de 4000 msnm son limitados. En las altas elevaciones las fluctuaciones del clima son menores, y por esa razón los estudios dendrocronológicos en combinación con datos de clima podrían aportar información confiable sobre la variabilidad climática (Álvarez et al., 2015; Beniston, 2003).

En Sudamérica se ha estudiado a Polylepis tarapacana, especie que se distribuye entre 3900 y $5200 \mathrm{~m}$ de altitud y con la que se ha reconstruido la precipitación y estudiado el impacto regional de ENSO (Argollo et al., 2004; Moya y Lara, 2011). En México se ha generado una red de información dendrocronológica del pino de altura (Pinus hartwegii Lindl.), especie que crece a 4000 msnm (Farjon et al., 1997; Villanueva et al., 2014b). Pero otras especies asociadas, como Juniperus monticola que alcanza 4300 msnm, no se han estudiado suficientemente y se desconoce su longevidad en diferentes regiones geográficas (Adams, 2014). Los estudios en otras regiones con Juniperus nana indican una longevidad de 334 años en Abisco, Suiza, mientras que Juniperus pingii subesp. Wilsonii en Nam Co, Tibet, China, alcanza 324 años de edad (Myers-Smith et al., 2015).

La forma arbustiva o rastrera con fuste retorcido de los individuos de Juniperus monticola lo definen como un "krummholz", un término alemán que refiere comunidades arbóreas enanas cuya función ecológica es capturar material orgánico y mineral transportado por el viento y depositarlo bajo el follaje para acelerar la formación de suelo en forma de islas (Körner, 2012).

El objetivo de este estudio fue analizar el potencial dendrocronológico de Juniperus monticola Martínez, mediante métodos dendrocronológicos tradicionales, bajo la hipótesis que los anillos presentes en Juniperus monticola son fechables, y que existe relación entre diferentes individuos en el área de estudio de tal forma que muestran una señal común (sincronía) en crecimiento que se explica por varia- bles del clima.

\section{MATERIALES Y MÉTODOS}

\section{Área de estudio}

El área de estudio, Monte Tláloc, se localiza en el municipio de Texcoco, Estado de México, con coordenadas $19.417^{\circ} \mathrm{N}$, y $98.715^{\circ} \mathrm{O}$ y una elevación de $4120 \mathrm{msnm}$ (Figura 1). El clima es frío, tipo $\mathrm{E}(\mathrm{T}) \mathrm{H}$ con temperatura media anual entre -2 y $5{ }^{\circ} \mathrm{C}$ (García, 2004). La precipitación media anual varía de 900 a $1200 \mathrm{~mm}$ (Figura 2).

\section{Secciones transversales}

Con el uso de una motosierra y sierras manuales se obtuvieron 50 rodajas de fustes o de ramas de Juniperus monticola con secciones relativamente cilíndricas, de árboles dispuestos en un transecto entre $3980 \mathrm{msnm}$ $\left(19.411^{\circ} \mathrm{N}, 98.719^{\circ} \mathrm{O}\right)$ y $4060 \mathrm{msnm}\left(19.417^{\circ} \mathrm{N}, 98.715\right.$ $\left.{ }^{\circ} \mathrm{O}\right)$ (Figura 1). El enebro presenta secciones transversales irregulares que influyen en el ancho de anillos en una dirección determinada. Esta condición implicó especial cuidado para buscar, hasta donde fuera posible, secciones regulares o circulares y en cada sección seleccionada considerar radios en varias direcciones hasta encontrar aquellos con la mayor intercorrelación. Lo anterior se logró verificando que el cofechado entre series mostrara correlación estadística con variables del clima (precipitación, temperatura); por ejemplo, los anillos anchos están asociados con años lluviosos y los angostos con periodos secos.

Las secciones transversales se secaron a temperatura ambiente y se pulieron con diversos grados de lija (granos 200 a 1200) para resaltar los anillos anuales. De cada sección transversal, se seleccionaron al menos tres radios a partir de su centro, como se sugiere para especies árticas y alpinas (Myers-Smith et al., 2015). Los crecimientos se midieron y fecharon mediante técnicas dendrocronológicas científicamente validadas (Fritts, 1976; Stokes y Smiley, 1968).

La calidad del fechado se corroboró mediante el programa COFECHA (Grissino-Mayer, 2001; Holmes, 1983), y el proceso de estandarización de los anchos de anillo anual se realizó con el programa ARSTAN (Cook, 1987) para verificar la sensibilidad media, inter-correlación media entre series y la señal expresada de la población (Briffa y Jones, 1990). La estandarización del anillo total se realizó con un modelo exponencial negativo, ya que las series de crecimiento mostraron una tendencia de "J invertida". Se empleó un "spline" de 128 años, con 50 \% de la varianza para ayudar a la eliminación de ruido no atribuido al clima (Cook y Holmes, 1984). 


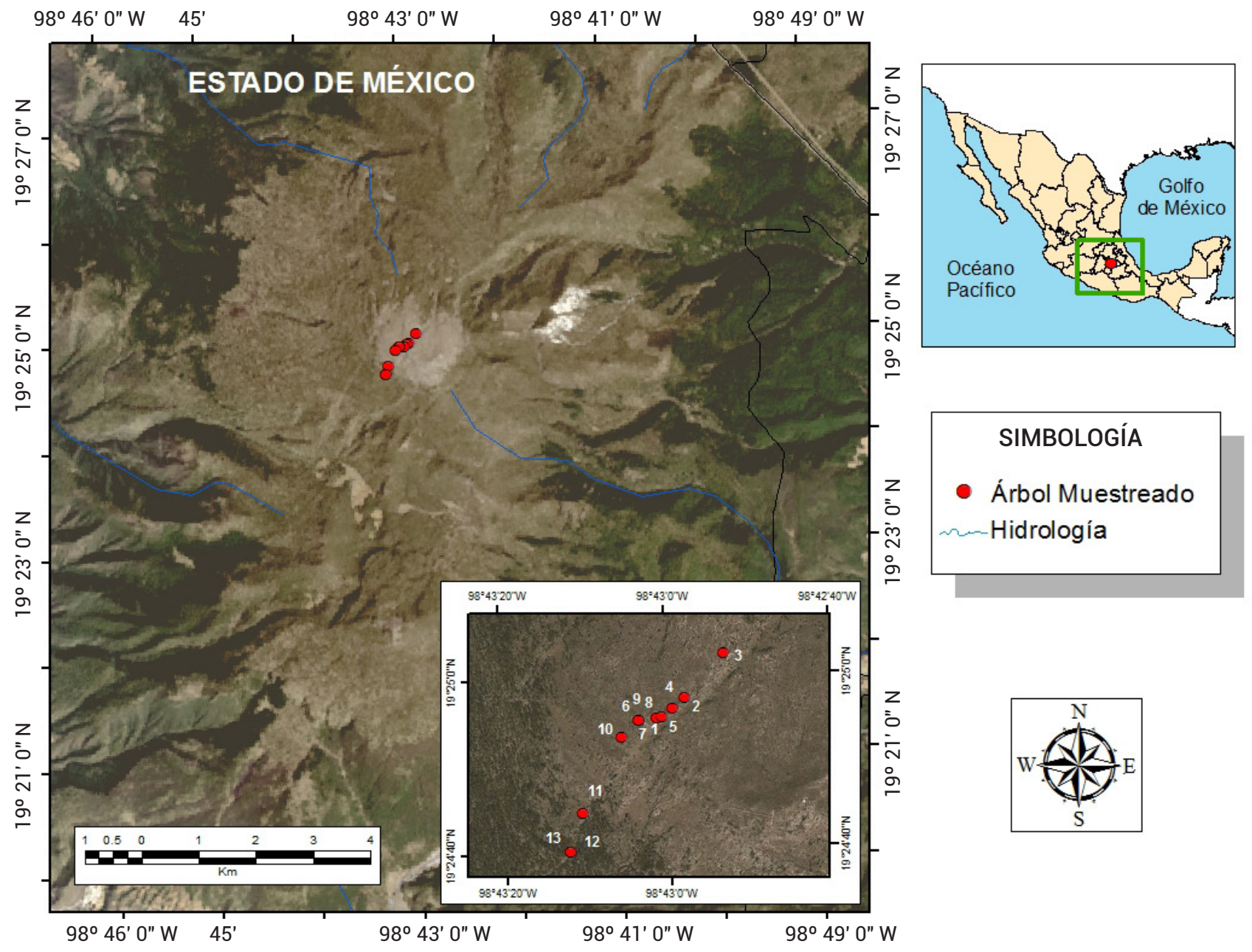

Figura 1. Localización del área de estudio y ubicación de los árboles muestreados. La imagen interior muestra la ubicación de los árboles muestreados.

Para valorar el potencial dendrocronológico de la especie estudiada, se tomó en cuenta la sensibilidad media que se refiere al cambio relativo en el grosor de los anillos de un año al siguiente; la inter-correlación, que mide la intensidad de la variación común entre series de crecimiento y es de utilidad para determinar si las series responden de manera similar a un factor ambiental como precipitación o temperatura. La señal expresada de la población (EPS, por sus siglas en inglés) es una medición de la calidad estadística de una cronología, que informa el grado en que ésta se asemeja a una cronología hipotética desarrollada con un número infinito de muestras; el valor de EPS tiene un rango de 0 a 1.0, donde un valor de 0.85 se considera aceptable (Brifa y Jones, 1990).

\section{Evaluación del potencial dendrocronológico}

Para evaluar este potencial se emplearon mediciones directas y estandarizadas de anchura de anillo anual de Juniperus monticola, y como referencia se emplearon series dendrocronológicas fechadas de Pinus hartwegii en rodales de los volcanes Iztaccíhuatl y Pico de Orizaba (Villanueva et al., 2014b).

Para analizar la respuesta climática de la especie y calcular la anualidad de sus crecimientos radiales, la serie dendrocronológica de anillo total de J. monticola se comparó con datos estimados de precipitación acumulada anual y de temperatura media anual del Monte Tláloc estimados por Franco-Plata et al. (2012). Así mismo, la cronología estándar se comparó con dos índices de variabilidad 


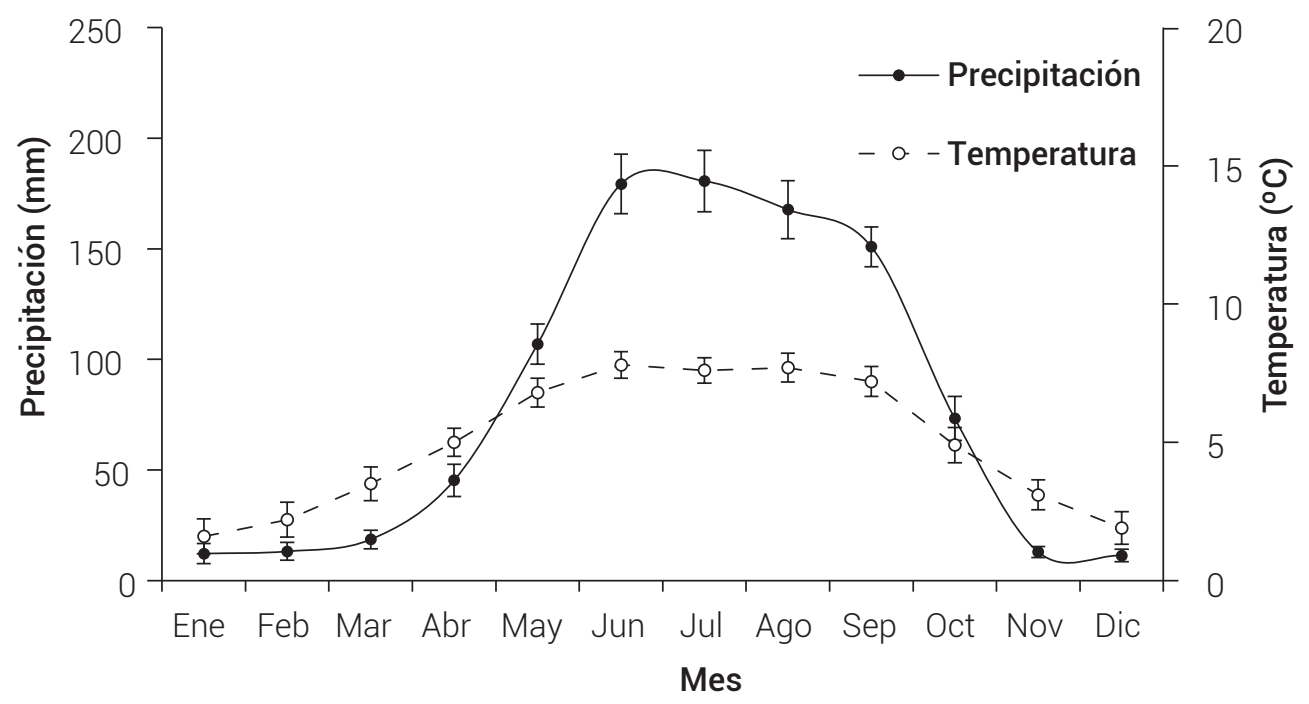

Figura 2. Precipitación y temperatura media mensual de cuatro estaciones meteorológicas cercanas al Monte Tláloc. Las barras verticales representan el error estándar. De acuerdo con la base de datos Eric III V2, la identificación de las estaciones meteorológicas corresponde a: 290, 1508, 1510 y 1521.

climática: (a) el índice de severidad de la sequía de Palmer (PDSI, por sus siglas en inglés) para el mes de junio, reconstruido para el centro de México (Stahle et al. 2012); el PDSI constituye un balance mensual hídrico que involucra datos de precipitación y temperatura, y es un indicador indirecto de la humedad actual del suelo (Cook et al., 2007). Y (b) el índice de oscilación del sur (SOI, por sus siglas en inglés), que se basa en la diferencia de presión atmosférica entre las islas de Tahití y Darwin, Australia, y mide la intensidad de la Circulación de Walker y de los eventos El Niño y La Niña para el periodo 1876-1996 (Allan et al., 1996).

Las series estandarizadas de ancho de anillo anual, la intensidad de una señal común y la correlación con series de Pinus hartwegii de los volcanes Iztaccíhuatl y Pico de Orizaba se emplearon para probar la hipótesis del potencial dendrocronológico de las especies. Aunque la correlación con SOI no es un indicador directo de potencial dendrocronológico, es un indicador climático que puede estar relacionado con el clima local y el crecimiento arbóreo.

\section{RESULTADOS}

\section{Fechado de las series}

Se logró fechar $66 \%$ de las series de crecimiento (33 de 50), que de acuerdo con COFECHA tuvieron un ancho de anillo anual promedio de $0.37 \mathrm{~mm}$, una desviación estándar promedio de $0.21 \mathrm{~mm}$, una inter-correlación entre series de $0.55(P<0.01)$ y una sensibilidad media de 0.41 . La cronología estándar de anillo total de J. monticola del
Monte Tláloc abarcó desde 1750 a 2013 (264 años), aunque el periodo más confiable para estudios climáticos acorde con el EPS de 0.85 se alcanzó con 10 radios para el periodo de 1810 a 2013 (Figura 3). A pesar de que algunas series estuvieron bien fechadas, tuvieron problemas de baja inter-correlación. La presencia de anillos falsos fue poco frecuente. El análisis ARSTAN indicó que la cronología media fue de 0.981, la sensibilidad media de 0.3023, desviación estándar de 0.2556, auto-correlación de primer orden de 0.46 , relación señal-ruido de 1.237, concordancia con la cronología de la población de 0.615 y varianza en el primer "eigenvector" de $54.83 \%$.

\section{Evaluación del potencial dendrocronológico}

El índice de ancho de anillo anual de Juniperus monticola y de Pinus hartwegii de las localidades de los volcanes Iztaccíhuatl y Pico de Orizaba mostraron correlaciones significativas $(P<0.01)$ de 0.26 y 0.40 , respectivamente. Principalmente, destacó la coincidencia de eventos extremos secos de las décadas de 1910, 1920, 1950, 1970 y 1990 (Figura 4). La variable estadísticamente correlacionada $(P<0.01)$ con el ancho de anillo total estandarizado fue la temperatura media anual (Figura 5). La variable precipitación no fue estadísticamente significativa.

\section{Correlación con índices de variabilidad climática (PDSI y SOI)}

Las series de índice de anillo anual de enebro se correlacionaron significativamente $(P<0.01)$ con los índices de 
sequía de severidad de Palmer (PDSI) reconstruido para el mes de junio en el centro de México y con el índice promedio anual de la oscilación del sur (SOI) (Figuras 6 y 7 ).

\section{DISCUSIÓN}

\section{Series fechadas}

Los valores de los parámetros de señal-ruido (1.24), sensibilidad media (0.41) y desviación estándar (0.49) indican que el enebro es viable para reconstrucciones paleoclimáticas. Al comparar con estudios en Pinus hartwegii (Villanueva-Díaz et al., 2014b; Yocom y Fulé, 2012;) y Pinus culminicola (Villanueva-Díaz et al., 2009b) que también crecen en regiones elevadas, el enebro muestra anillos anuales más estrechos $(0.21 \mathrm{~mm})$ y menor señal climática común, pero su cambio relativo de un año a otro (0.41) es mayor que el de esas otras dos especies (Cuadro 1). Con relación a la longevidad de $\mathrm{J}$. monticola se encontraron individuos cercanos a los 300 años de edad, que son jóvenes si se considera que en algunos volcanes del centro de México se han encontrado individuos de 400 años en el Iztaccíhuatl y de hasta 900 años en el Cofre de Perote y Pico de Orizaba (Villanueva-Díaz, Com. pers. ${ }^{1}$ ).

La sensibilidad media de 0.41 determinada para enebro es relativamente alta si se toma en cuenta que crece en condiciones adversas de suelos someros y rocosos (Farjon, 2013; Giménez de Azcárate y Escamilla, 1999) y la irregularidad en crecimiento en los diversos radios del fuste. Las especies del norte de México como Pseudotsuga menziesii, que crecen en ambientes más benignos, presentan valores de sensibilidad media de 0.13 a 0.40 (Villanueva-Díaz et al., 2008). La sensibilidad media más alta en especies mexicanas corresponde a las de climas secos como Pinus cembroides y Pinus pinceana con valores de hasta 0.7

José Villanueva-Díaz. Especialista en dendrocronología y encargado del Laboratorio Nacional de Dendrocronología en México. INIFAP CENID-RASPA, en Gómez Palacio, Durango.
(Constante-García et al., 2010; Santillán et al., 2010).

\section{Evaluación del potencial dendrocronológico}

La correlación encontrada entre series de anillos de $P$. hartwegii y J. monticola es baja pero significativa. Es posible que esto se deba a que los periodos de actividad del cambium no sean similares entre especies. En otros estudios en los que se ha comparado la correlación de anillos entre especies que crecen en lugares cercanos, la correlación es de hasta 0.78; sin embargo, las correlaciones con el clima en general son menores a 0.6 (Maxwell et al., 2015).

La correlación significativa $(P<0.01)$ de la serie dendrocronológica generada para los últimos 108 años (1900 a 2007) con la especie Pinus hartwegii en el Iztaccíhuatl y el Pico de Orizaba (Figura 4), corrobora el potencial del enebro para fines dendroclimáticos. La coincidencia de las series es mayor al analizar periodos muy secos, como los ocurridos en las décadas de 1910, 1920, 1950, 1970 y 1990, donde los índices estandarizados de J. monticola coinciden en general con los de las series dendrocronológicas de P. hartwegii. Este comportamiento se evidencia también en años húmedos cuando los valores estandarizados para J. monticola en general son más altos, lo cual muestra su sensibilidad climática. La correspondencia observada entre los índices dendrocronológicos de Juniperus monticola y Pinus hartwegii es una confirmación de que los anillos de la especie son anuales y que, por su alta sensibilidad a la temperatura, pueden ser utilizados con fines de reconstrucción paleoclimática.

La presencia de anillos falsos en Juniperus monticola fue de baja frecuencia, como se ha encontrado para otras especies de hábitat alpino (Liang y Eckstein, 2009). La baja frecuencia de anillos falsos en esta especie se puede explicar por lo siguiente: (1) La estacionalidad es marcada y da lugar a un anillo anual estrecho de 0.2 a $0.5 \mathrm{~mm}$; (2) La

Cuadro 1. Parámetros estadísticos de las cronologías de Juniperus montícola y otras especies que habitan sitios de alta montaña en el centro y norte del país.

\begin{tabular}{llcccccc}
\hline \multirow{2}{*}{ Especie } & \multirow{2}{*}{ Sitio } & \multirow{2}{*}{ Ancho del anillo $(\mathrm{mm})$} & \multicolumn{4}{c}{ Serie estandarizada } \\
\cline { 5 - 8 } & & & IAA & SM & DE $(\mathrm{mm})$ & AC1 & RSR \\
\hline Juniperus monticola & Monte Tláloc, Edo. Méx. & 0.37 & 1.0 & 0.41 & 0.49 & 0.42 & 1.24 \\
Pinus hartwegii & Iztaccíhuatl, Edo. Méx & 1.74 & 0.99 & 0.36 & 0.42 & 0.49 & 3.2 \\
Pinus culminicola & Cerro Potosí, N. L. & \multirow{2}{*}{0.79} & 0.99 & 0.28 & 0.35 & 0.37 & 5.5 \\
\hline
\end{tabular}

DE: Desviación estándar: SM: sensibilidad media, medición del cambio relativo en el grosor de los anillos de un año con relación al siguiente crecimiento en una serie de mediciones; IAA: índice medio de ancho de anillo, derivado del proceso de estandarización y generalmente se obtiene al dividir el valor de medición entre el valor de la curva; AC1: auto-correlación de primer orden, que mide la influencia en crecimiento que tiene el año previo con relación al crecimiento del año actual; RSR: relación Señal-Ruido, que muestra el grado o intensidad en que las series captan la señal climática común, entre mayor es el valor de la relación su potencial es mayor para fines de reconstrucción climática. Una señal expresada de la población (EPS por sus siglas en inglés) de 0.85 se alcanzó con 10 núcleos de crecimiento, a partir del año 1810. 
precipitación a esa elevación normalmente no es un factor limitante, por lo que difícilmente se forman bandas falsas de crecimiento; 3) El programa COFECHA considera indirectamente la posibilidad de anillos falsos mediante la falta de correlación o desfase de las series entre muestras independientes, y éste no fue el caso; y 4) Ocasionalmente se observaron posibles bandas obscuras en un anillo anual, pero anatómicamente no se mostraron como las bandas tenues que caracterizan a un anillo falso.

La correlación significativa de la serie dendrocronológica de J. monticola con la temperatura media anual $(r=0.45$, $P<0.01$ ), indica que esta variable explica $20 \%$ del crecimiento radial de la especie en cuestión (Figura 5). Este hallazgo es relevante para estudios de cambio climático, ya que las especies hasta ahora utilizadas en investigaciones dendroclimatológicas en México en general responden a variaciones de la precipitación más que de temperatura (Cleaveland et al., 2003; Meko et al., 2013; Villanueva-Díaz et al., 2007).

La sensibilidad de la especie a índices que involucran en forma combinada temperatura y humedad disponible se verificó al comparar los índices de ancho de anillo anual y el de severidad de la sequía (PDSI) reconstruido para el mes de junio en el centro de México (Stahle et al., 2012). Se determinó una asociación significativa entre ambas variables $(r=0.25, P<0.01)$ para el periodo común 19002008, donde el crecimiento de la especie fue favorecido por valores menos extremos (positivos) del PDSI, es decir por condiciones más benignas de temperatura y humedad; sin embargo, sólo se explica $6.3 \%$ de la variabilidad de los anillos (Figura 6). Por otra parte, al explorar la correlación por periodos de 30 años, la correlación significativa solo se sostiene a principios y finales del siglo pasado, con $r=0.53$ $(P<0.01)$ de 1900 a 1929; y de $0.55(P<0.01)$ de 1990 a 2008; en el tiempo intermedio de esos periodos se pierde la significancia estadística.

A pesar de que se pudo establecer una relación positiva con indicadores combinados de humedad temperatura y el ancho del anillo anual, la mayor explicación de la variación de los anillos fue en la temperatura media como variable independiente (20\%). Esta información es congruente al comparar los valores anuales del SOI con la cronología de anillo total, de la que resultó una asociación significativa, pero negativa $(r=-0.27)$; es decir que en eventos intensos de El Niño (valor más negativo), que corresponden con menor precipitación y mayor temperatura en el centro de México (Magaña et al., 2003), los anillos fueron más anchos, situación asociada a un incremento en las temperaturas que parece favorecer el crecimiento de esta especie (Figura 7). Este resultado indica que los anillos del enebro son útiles para la reconstrucción de la temperatura media anual, y que a diferencia de otras especies un aumento en temperatura estimula su crecimiento radial.

\section{Correlación con índices de variabilidad climática (PDSI y SOI)}

La relación entre el crecimiento y el PDSI, este último también afectado por la presencia de ENSO en su fase Niño para el centro de México, indicó que los mayores crecimientos ocurrieron en años catalogados como Niño intenso; casos específicos los de $1930(1.11,-2.48), 1939$ (1.10,-1.34), 1946 (1.1503, -2.25), $1957(1.50,-0.23), 1958$ $(1.54,-0.16), 1982(1.60,-2.11), 1983(1.24,-4.19)$ y 2004 (1.08, -0.03), en donde los valores entre paréntesis constituyen el índice de ancho de anillo y el valor anual del SOl, respectivamente.

El enebro del centro de México muestra inter-correlación alta (0.52), como las especies alpinas de Sudamérica y de Asia. Por ejemplo, la inter-correlación de Polylepis tarapacana en los Andes varía de 0.26 a 0.36 (Argollo et al., 2004), mientras que para Rhododendrom nivale en la meseta del Tibet fue de 0.1 (Liang y Eckstein, 2009). En el caso de Nothofagus pumilio en el límite altitudinal arbóreo de los Andes, la inter-correlación fue de 0.48 y la variación de las series se explicó por la temperatura principalmente (Álvarez et al., 2015). La reducción en la inter-correlación entre sitios y especies se explica por secuencias erráticas de liberación y compresión de crecimiento radial (Liang y Ecktein, 2009; Myers-Smith et al., 2015) que influyen en una distribución irregular de las hormonas de crecimiento (Schweingruber, 1996).

La correlación negativa entre el índice de ancho de aniIlo (RWI) y el índice de oscilación del sur (SOI) indica que los valores altos de crecimiento radial corresponden con valores bajos de SOI. Esta situación también se reflejó con el PDSI, que indicó mayores incrementos radiales con valores bajos (condiciones más secas). Por otro lado, este resultado es consistente con el impacto de ENSO, particularmente en años catalogados como de Niño intenso.

La tendencia a un incremento en la temperatura en Monte Tlaloc a $4000 \mathrm{msnm}$, de 5.35 a $6.45^{\circ} \mathrm{C}$ en los últimos 50 años (Franco-Plata et al., 2012), parece relacionarse con el incremento en crecimiento radial de Juniperus monticola, corroborado por la asociación significativa entre ambas variables y de manera más notoria en los últimos 15 años (Figura 7). El impacto de un incremento en la temperatura atribuido al calentamiento global y su influencia en el crecimiento radial de especies arbóreas, ha sido analizado mediante modelos predictivos de cambio climático, con efectos diferentes dependiendo del hábitat de las especies analizadas; de esta manera, se ha encontrado una relación 


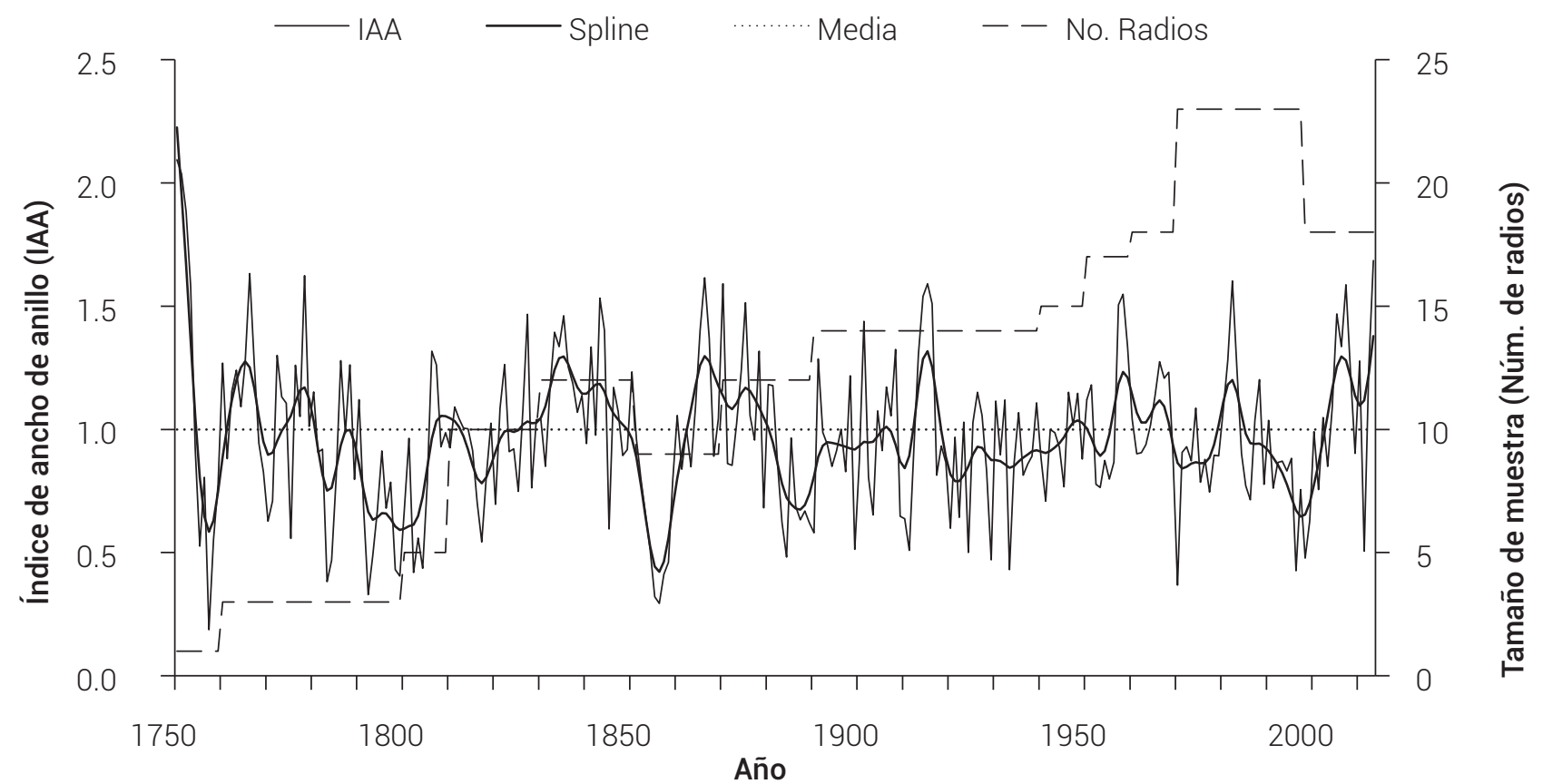

Figura 3. Serie dendrocronológica estandarizada de ancho de anillo total de Juniperus monticola en el Monte Tláloc, Texcoco, Estado de México. La cronología tiene una extensión de 264 años (1750-2013). La línea sólida en gris corresponde a los índices anuales; la línea sólida en negro es una línea suavizada a nivel de década, para resaltar eventos de baja frecuencia; la línea horizontal punteada corresponde al valor medio de los índices, que es cercano a 1.0. La señal expresada de la población (EPS) se alcanzó con 8 árboles.

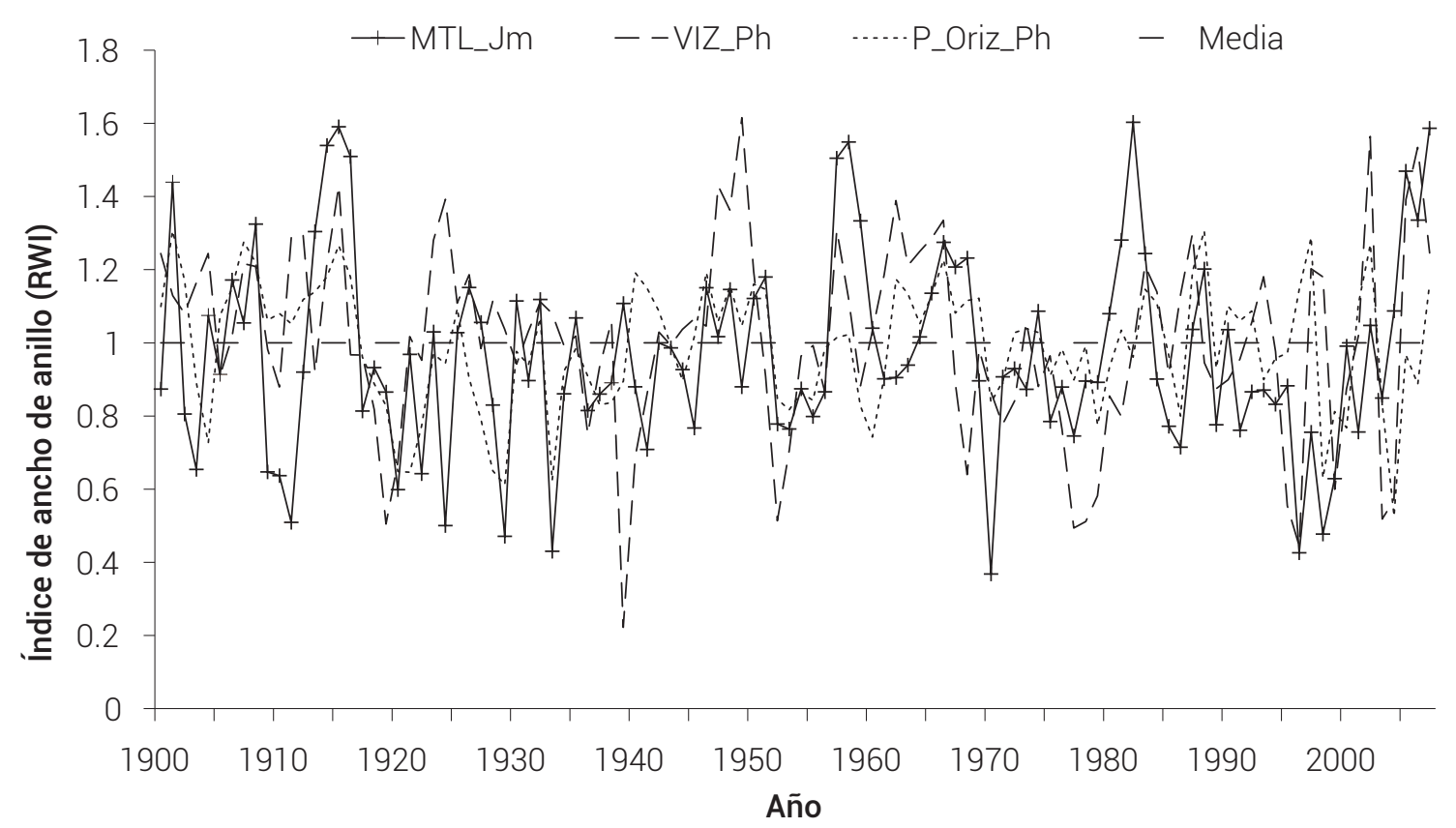

Figura 4. Comportamiento de los índices de crecimiento de Juniperus monticola y de Pinus hartwegii, especies que constituyen, respectivamente, los límites superiores de la vegetación leñosa y de la vegetación arbórea en volcanes del centro de México. MTL_Jm: Juniperus monticola, Monte Tláloc; VIZ_Ph: Pinus hartwegii, Iztaccíhuatl; P_Oriz_Ph: Pinus hartwegii, Pico de Orizaba. 


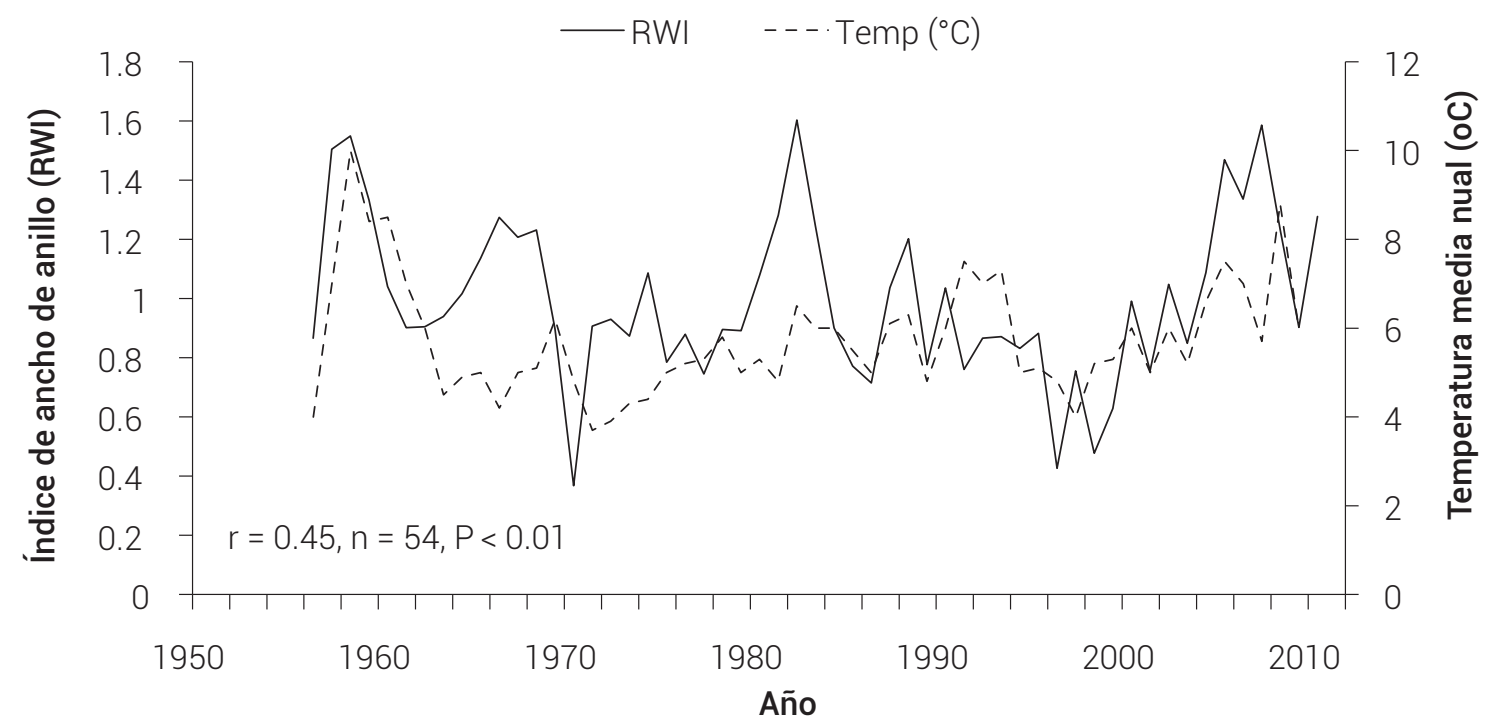

Figura 5. Relación entre la cronología estandarizada de ancho de anillo total de Juniperus monticola (RWI) y la temperatura media anual (Temp ${ }^{\circ} \mathrm{C}$ ) para Monte Tláloc, Edo. de México, con datos climáticos reportados por Franco-Plata et al. (2012).

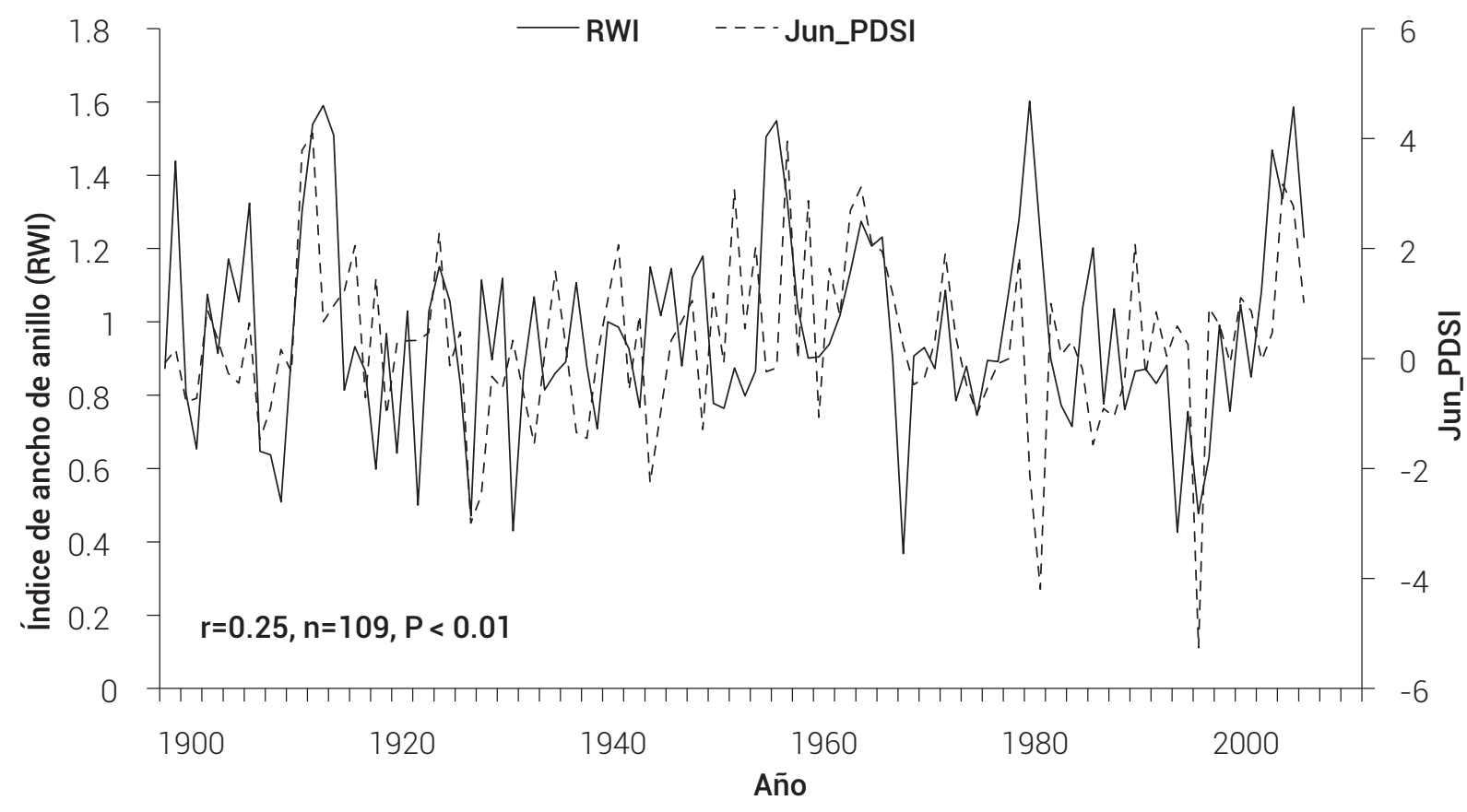

Figura 6. Relación entre los índices estandarizados de crecimiento de J. monticola del Monte Tláloc (RWI) y el índice de sequía de severidad de Palmer reconstruido para el mes de junio en el centro de México (Jun_PDSI; Stahle et al., 2011). 


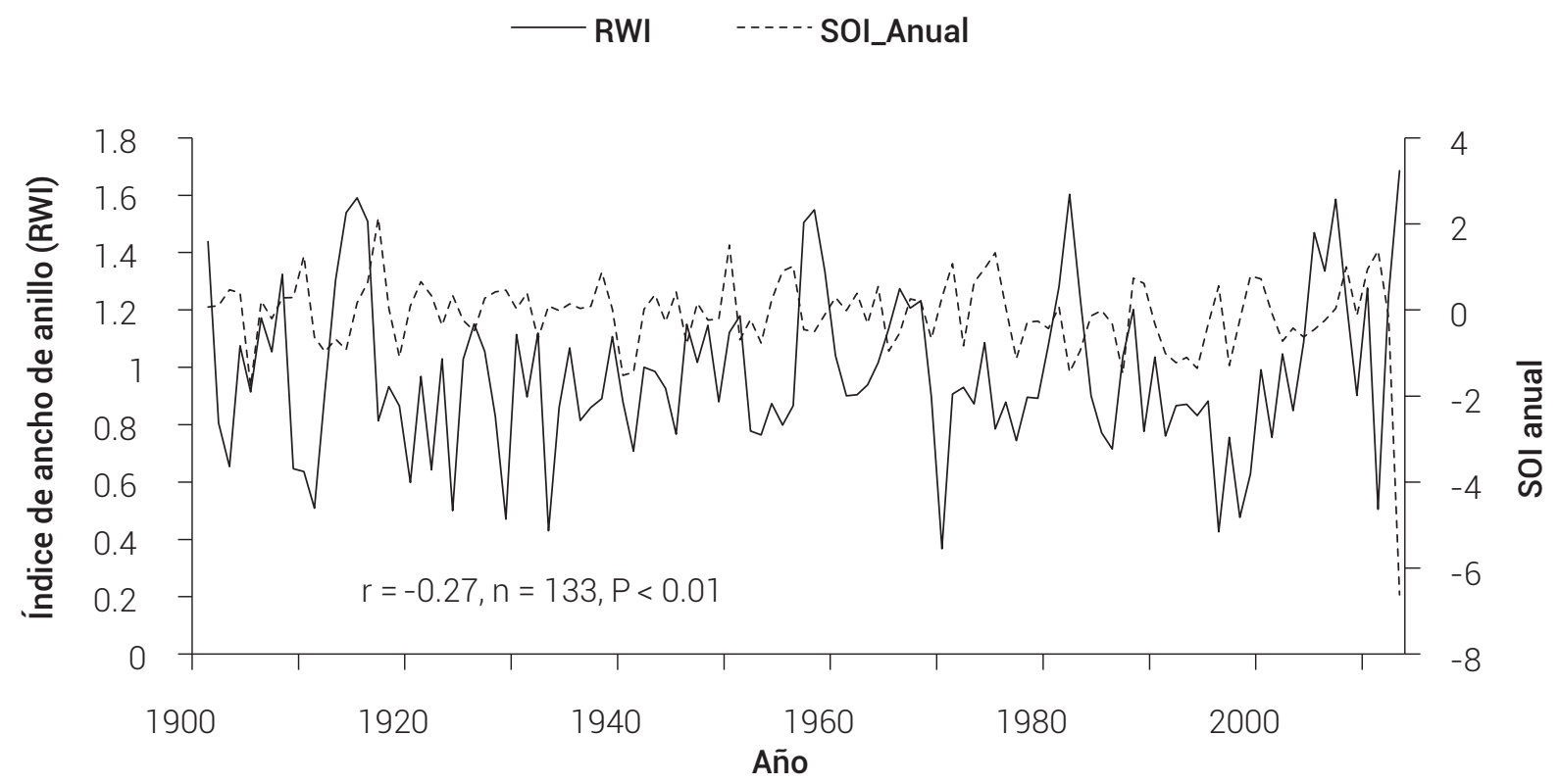

Figura 7. Relación entre el índice promedio anual de la Oscilación del Sur (SOI_Anual; Allan et al., 1996) y la cronología de anillo total de J. montícola del Monte Tláloc (RWI). La relación es negativa, lo que implica un efecto favorable de condiciones El Niño en el crecimiento del enebro.

positiva entre el incremento en temperatura y el crecimiento de especies de coníferas en zonas frías en el norte de Europa (Tishin y Chizhikova, 2013). Sin embargo, en bosques de Pinus hartwegii en México se han encontrado reducciones en crecimiento, debido tanto a un incremento de la temperatura como a una disminución en la precipitación en picos elevados de México (Ricker et al., 2007).

Pocos estudios, sin embargo, han analizado la relación directa entre variables climáticas y el incremento radial; uno de esos contados estudios se desarrolló cerca del límite arbóreo del Nevado de Colima, donde se midió el inicio y fin de la estación de crecimiento para $P$. hartwegii y la influencia de diversas variables climáticas procesadas en tiempo real por una estación climática automatizada (Biondi et al., 2005). La generación de series dendrocronológicas de especies de alta montaña, como es el caso de Juniperus monticola, puede contribuir a un mejor entendimiento de la variabilidad climática y de su impacto en los ecosistemas alpinos (Myers-Smith et al., 2015).

El potencial dendroclimático de una especie depende de su sensibilidad, pero también de su longevidad. Los muestreos en tres montañas del centro de México hasta ahora indican que $\mathrm{J}$. monticola es una especie con individuos longevos que pueden superar los 800 años de edad, lo cual permite considerarla como una de las especies más longevas -probablemente la mayor- hasta ahora encontrada en las montañas altas del centro de México (VillanuevaDíaz et al., 2010).

\section{CONCLUSIONES}

Los parámetros dendrocronológicos de calidad del fechado y estandarización indicaron que Juniperus monticola es viable para estudios dendrocronológicos. Los anillos de esta especie son anuales, sensibles a la variabilidad climática, como lo indica la inter-correlación entre series y una señal de crecimiento común. La correlación significativa de las series de Juniperus monticola con series fechadas de Pinus hartwegii y temperatura media anual confirman el potencial dendrocronológico del enebro para reconstruir la variabilidad de temperatura en elevaciones mayores de 4000 msnm. La correlación significativa de la serie dendrocronológica de J. monticola con los índices severidad de la sequía de Palmer y oscilación del sur (PDSI y SOI) demuestra que los fenómenos geográficos de clima se registran en anillos de especies alpinas de México.

\section{AGRADECIMIENTOS}

La presente investigación se apoyó con fondos de los proyectos Programa UNAM-DGAPA-PAPIIT clave IN105213 y Estudio de la relación clima-incendios en el Norte-Centro de México fondo sectorial SEP-CONACT, proyecto 136223. 


\section{BIBLIOGRAFÍA}

Adams R. P. (2014) Junipers of the World: The genus Juniperus. Trafford Publishing. Bloomington, IN. E.U.A. 422 p.

Allan R., J. Lindesay and D. Parker (1996) El Niño: Southern oscillation and climatic variability. CSIRO Publishing, Atmospheric Research, Australian National University. United Kingdom. 408 p.

Álvarez C., T. T. Veblen, D. A. Christie and A. González-Reyes (2015) Relationships between climate variability and radial growth of Nothofagus pumilio near altitudinal treeline in the Andes of northern $\mathrm{Pa}$ tagonia, Chile. Forest Ecology and Management 342:112-121.

Argollo J., C. Soliz y R. Villalba (2004) Potencialidad dendrocronológica de Polylepis tarapacana en los Andes Centrales de Bolivia. Ecología en Bolivia 39:5-24.

Beniston M. (2003) Climatic change in mountain regions: A review of possible impacts: In: Climate Variability and Change in High Elevation Regions: Past, Present \& Future. Springer, Netherlands. pp:5-31.

Biondi F., A. Gershunov and D. R. Cayan (2001) North Pacific decadal climate variability since 1661. Journal of Climate 14:5-10.

Biondi F., P. C. Hartsough and I. G. Estrada (2005) Daily weather and tree growth at the tropical treeline of North America. Artic, Antartic, and Alpine 37:16-24.

Briffa K. R. and P. D. Jones (1990) Basic chronology statistics and assessment In: Methods of Dendrochronology: Applications in the Environmental Sciences. E. R. Cook and L. A. Kairiukstis (eds.). Kluwer Academic Publishers. Boston. pp:137-152.

Brito-Castillo L., S. D'ıaz-Castro, C. A. Salinas-Zavala and A. V. Douglas (2003) Reconstruction of long-term winter streamflow in the Gulf of California continental watershed. Journal of Hydrology 278:3950.

Constante-García V., J. Villanueva-Díaz, J. Cerano-Paredes y J. Estrada-Ávalos (2010) Parámetros para definir el potencial dendrocronológico. INIFAP-CENID-RASPA. Gómez Palacio, Dgo. 40 p.

Cook E. R. (1987) The decomposition of tree-ring series for environmental studies. Tree-Ring Bulletin 47:37-59.

Cook E. R. and R. L. Holmes (1984) Program ARSTAN user's manual. Laboratory of Tree-Ring Research. University of Arizona. Tucson, AZ. 15 p.

Cook E. R., R. Seager, M. A. Cane and D. W. Stahle (2007) North American drought: Reconstructions, causes, and consequences. EarthScience Reviews 81:93-134

Correa-Díaz A., A. Gómez-Guerrero, J. Villanueva-Díaz, L. U. Castruita-Esparza, T. Martínez-Trinidad y R. Cervantes-Martínez (2014) Análisis dendroclimático de ahuehuete (Taxodium mucronatum Ten.) en el centro de México. Agrociencia 48:537-551.

Cleaveland M. K., D. W. Stahle, M. D. Therrell, J. Villanueva-Díaz and B. T. Burns (2003) Tree-ring reconstructed winter precipitation and tropical teleconnections in Durango, Mexico. Climatic Change 59:369388.

Díaz S. C., R. Touchan and T. W. Swetnam (2001) A tree-ring reconstruction of past precipitation for Baja California Sur, Mexico. International Journal of Climatology 21:1007-1019.

Farjon A., J. A. P. de la Rosa and B. T. Styles (1997) A Field Guide to the Pines of Mexico and Central America. Royal Botanic Gardens. United Kingdom. $147 \mathrm{p}$.

Farjon A. (2013) Juniperus monticola. The IUCN Red List of Threatened Species. Version 2014.2. www.iucnredlist.org (September 2014).

Franco-Plata R., M. A. Gómez-Albores, L. R. Manzano-Solís y C. Díaz-Delgado (2012) La naturaleza frente al hombre: el clima, condiciones actuales y variabilidad espacio-temporal. In: Gobierno del Estado de México y Universidad Autónoma del Estado de México. Monte Tláloc II, la casa del Dios del Agua. Dirección de Difusión y Promoción de la Investigación y los Estudios Avanzados, SIEA, UAEM. México. pp:29-48.

Fritts H. C. (1976) Tree Rings and Climate. Academic Press. New York. $567 p$.

García E. (2004) Modificaciones al sistema de clasificación climática de Köpen (Para adaptarlo a las condiciones de la República Mexicana). Instituto de Geografía UNAM. México D.F. 98 p.

Giménez de Azcárate J. y M. Escamilla (1999) Las comunidades edafoxerófilas (enebrales y zacatonales) en las montañas del centro de
México. Phytocoenologia 29:449-468

Grace J., F. Berninger and L. Nagy (2002) Impacts of climate change on the tree line. Annals of Botany 90:537-544.

Grissino-Mayer H. D. (2001) Evaluating crossdating accuracy: A manual and tutorial for the computer program COFECHA. Tree-Ring Research 57:205-221

Holmes R. L. (1983) Computer assisted quality control in tree ring dating and measurement. Tree Ring Bulletin 44:69-78.

Körner C. (2012) Alpine treelines: Functional ecology of the global high elevation tree limits. Springer. Basel, Switzerland. 220 p.

Liang E. and D. Eckstein (2009) Dendrochronological potential of the alpine shrub rhododendron nivale on the south-eastern Tibetan Plateau. Annals of Botany 104:665-670

Magaña V. O., J. L. Vázquez, J. L. Pérez y J. B. Pérez (2003) Impact of El Niño on precipitation in Mexico. Geofísica Internacional 42:313-330.

Maxwell J. T., G. L. Harley and T. J. Matheus (2015) Dendroclimatic reconstructions from multiple co-occurring species: A case study from an old-growth deciduous forest in Indiana, USA. International Journal of Climatology 35:860-870

Meko D., R. Touchan, J. V. Díaz, D. Griffin, C. Woodhouse, C. Castro, C. CarriIlo and S. Leavitt (2013) Sierra San Pedro Mártir, Baja California, cool season precipitation reconstructed from earlywood width of Abies concolor tree rings. Journal of Geophysical Research: Biogeosciences 118:1660-1673.

Moya J. y A. Lara (2011) Cronologías de ancho de anillos de queñoa (Polylepis tarapacana) para los últimos 500 años en el altiplano de la región de Arica y Parinacota, Chile. Bosque (Valdivia) 32:165-173

Myers-Smith I. H., M. Hallinger, D. Blok, U. Sass-Klaassen, S. A. Rayback S. Weijers, A. J. Trant, K. D. Tape, A. T. Naito, S. Wipf, C. Rixen, M. A. Dawes, J. A. Wheeler, A. Buchwal, C. Baittinger, M. Macias-Fauria, B. C. Forbes, E. Lévesque, N. Boulanger-Lapointe, I. Beil, V. Ravolainen and M. Wilmking (2015) Methods for measuring arctic and alpine shrub growth: A review. Earth-Science Reviews 140:1-13.

Pompa-García M., L. Miranda-Aragón and C. A. Aguirre-Salado (2015) Tree growth response to ENSO in Durango, Mexico. International Journal of Biometeorology 59:89-97.

Ricker M., G. Gutiérrez-García and D. C. Daly (2007) Modeling long-term tree growth curves in response to warming climate: Test cases from a subtropical mountain forest and a tropical rainforest in Mexico. Canadian Journal of Forest Research 37:977-989.

Santillán-Hernández M., E. H. Cornejo-Oviedo, J. Villanueva-Díaz, J. CeranoParedes, S. Valencia-Manzo and M. A. Capó-Arteaga (2010) Potencial dendroclimático de Pinus pinceana Gordon en la Sierra Madre Oriental. Madera y Bosques 16:17-30.

Schweingruber F. H. (1996) Tree Rings and Environment: Dendroecology. Paul Haupt AG Bern. Switzerland. 609 p.

Seager R., M. Ting, M. Davis, M. Cane, N. Naik, J. Nakamura, C. Li, E. Cook and D. W. Stahle (2009) Mexican drought: An observational modeling and tree ring study of variability and climate change. Atmósfera 22:1-31

Stahle D. W., D. J. Burnette, J. V. Díaz, R. R. Heim, Jr. F. K. Fye, J. C. Paredes, R. A. Soto and M. K. Cleaveland (2012) Pacific and Atlantic influences on Mesoamerican climate over the past millennium. Climate Dynamics 39:1431-1446

Stokes M. A. and T. L. Smiley (1968) An Introduction to Tree-ring Dating. Chicago, IL. USA. $73 p$

Tishin D. and N. Chizhikova (2013) Detection of plausible radial growth scenarios for Pinus sylvestris L. responding to the climate change at the north of the east European plain. Open Forest Science Journal 6:57-59.

Villanueva-Díaz J., D. W. Stahle, B. H. Luckman, J. Cerano-Paredes, M. D. Therrell, M. K. Cleaveland and E. Cornejo-Oviedo (2007) Winter-spring precipitation reconstructions from tree rings for northeast Mexico. Climatic Change 83:117-131.

Villanueva-Díaz J., J. Cerano-Paredes, D. W Stahle, J. Estrada-Ávalos y V. Constante-García (2008) Potencial Dendrocronológico de Pseudotsuga menziesii (Mirb.) Franco y Reconstrucciones de Precipitación y Flujo en México. Folleto Científico No. 23. INIFAP CENID-RASPA. Gómez Palacio, Durango. 49 p.

Villanueva-Díaz J., J. Cerano-Paredes, D. W. Stahle, V. Constante-García y J. Estrada-Ávalos (2009a) Potencial dendrocronológico del Pino Potosí (Pinus culminicola) especie endémica de la Sierra Madre 
Oriental. Memoria in extenso de la XXI Semana Internacional de Agronomía FAZ-UJED, Gómez Palacio, Dgo. pp:1004-1007.

Villanueva Díaz J., P. Z. Fulé, J. Cerano-Paredes, J. EstradaÁvalos and I. Sánchez-Cohen (2009b) Reconstrucción de la precipitación estacional para el barlovento de la Sierra Madre Occidental con anillos de crecimiento de Pseudotsuga menziesii (Mirb.) Franco. Ciencia Forestal en México 34:37-69.

Villanueva-Díaz J., J. Cerano-Paredes, D. W. Stahle, V. Constante-García, L. Vázquez-Salem, J. Estrada-Ávalos y J. D. Solorio-Benavides (2010) Árboles longevos de México. Revista Mexicana de Ciencias Forestales 1:7-30

Villanueva Díaz J., J. Cerano P., D. W. Stahle, B. H. Luckman, M. D. Therrell, M. K. Cleaveland, y P. Z. Fulé (2011) La dendrocronología y reconstrucciones paleoclimáticas en el Norte-Centro de México. In: Escenarios de Cambio Climático. M. Caballero y B. Ortega G. (comps.). Registros del Cuaternario en América Latina I. UNAM Instituto de Geofísica, Dirección General de Publicaciones y Fo- mento Editorial. México, D.F. pp:47-72.

Villanueva-Díaz J., J. Cerano-Paredes, A. Gómez-Guerrero, A. Correa-Díaz, L. U. Castruita-Esparza, R. Cervantes-Martínez, D. W. Stahle y A. R. Martínez-Sifuentes (2014a) Cinco siglos de historia dendrocronológica de los ahuehuetes (Taxodium mucronatum Ten.) del parque El Contador, San Salvador Atenco, Estado de México. Agrociencia 48:725-737.

Villanueva-Díaz J., J. Cerano-Paredes, L. Vázquez-Selem, D. W. Stahle, P. Z. Fulé, L. L. Yocom, O. Franco-Ramos y J. A. Ruiz-Corral (2014b) Red dendrocronológica del pino de altura (Pinus hartwegii Lindl.) para estudios dendroclimáticos en México. Investigaciones Geográficas, Boletín del Instituto de Geografía. doi: 10.14350/ rig.42003.

Yocom L. L. and P. Z. Fulé (2012) Human and climate influences on frequent fire in a high elevation tropical forest. Journal of Applied Ecology 49:1356-1364. 